\title{
HERBERT CANNULATED BONE SCREW OSTEOSYNTHESIS VERSUS MINIPLATES OSTEOSYNTHESIS IN THE TREATMENT OF MANDIBULAR ANGLE FRACTURES
}

\author{
Haytham Al-Mahalawy*
}

\begin{abstract}
Purpose: Mandibular angle fractures are commonly encountered in the maxillofacial field, their surgical treatment includes a wide range of fixation techniques. Herbert cannulated bone screw is a successful minimally invasive mean of fracture fixation that is used routinely in orthopedic surgery. The aim of this study was to evaluate the clinical and radiographic performance of a solitary Herbert bone screw in the treatment of mandibular angle fractures and compare it with the commonly established treatment modality with double 2.0 - $\mathrm{mm}$ miniplates.
\end{abstract}

Patients and methods: Twenty patients, 15 males and 5 females,with unilateral isolated fracture in the mandibular angle region were selected and randomly allocated into the following groups: group I $(n=10)$ treated with a solitary Herbert Bone Screw and group II $(n=10)$ treated with doubleminiplates. Patients were clinically and radiographically monitored for twelve weeks.

Results:The occlusal examination showed a normal occlusion in all of the cases in both groups. Both groups showed a statistically significant gain in the mean bone density across the follow-up period $(P<0.001)$. The difference between groups was statistically insignificant $(P=0.761)$.

Conclusions: The utilization of a solitary Herbert cannulated bone screw osteosynthesis provides a successful, more economic, minimally invasive and predictable treatment modality for the treatment of mandibular angle fractures.

\section{INTRODUCTION}

Mandibular fractures comprise between 40 and $62 \%$ of all facial fractures, making them the second most commonly encountered facial fractures following nasal bone fractures ${ }^{1}$. The thin bony junction and the presence of the third molar make the angle of the mandible one of the most prone areas to fractures, they account for 25 to $35 \%$ of all mandibular fractures ${ }^{2}$.

Mandibular angle fractures (MAFs) may result from direct lateral trauma or indirect chin or contralateral parasymphyseal area trauma. Furthermore, their prognosis may be judged as favorable or unfavorable depending on the degree

* Lecturer, Oral and Maxillofacial Surgery Department, Faculty of Dentistry, Fayoum University, Fayoum, Egypt. 
of displacement, the direction of the fracture line and vector of muscle pull force ${ }^{3}$.

Owing to the significant possibility of postoperative complication after MAFs management, several treatment modalities were proposedusing various forms of plates and screws systems. However, the choice of one modality over the other depends onseveral diverse variables such as the degree of fragments displacement, fragments comminution, patient age, socioeconomic variables and surgeons experience and preference ${ }^{4}$.

Anabundant amount of discussion has arousedconcerning the most relevant treatment modality for MAFs since the introduction of miniplates into the maxillofacial trauma. Champy et al $(1978)^{5}$ assessed the anatomical and biomechanical consideration for placing miniplates and came out with the concept of ideal lines of osteosynthesis, where the application of these miniplates can be limited to the tension band on the upper border ${ }^{6}$. However, several authors demonstrate the superiority of double miniplates where they exhibit lower mechanical stresses ${ }^{7-9}$.

Traction osteosynthesis based on axial compression of the bone fragments which was introduced in the maxillofacial region by Brons and Boeringin $1970^{10}$. A recent development was introduced where only a solitary lag screw is used for fixation of $\mathrm{MAFs}^{11}$.This edges plate osteosynthesis in providing more firm fracture stabilization which in turn support rapid primary healing while at the same time reduces the required amount of hardware, tissue exposure and operative time ${ }^{10}$. Nonetheless, the lag screw spherical head exerts high pressure on a very small area of bone, acting like a wedge, which increases the probability of bone cracking and damage. This may be decreased using a countersink drill, which is difficult to use in the angle region, or combining the screw head with a biconcave washer ${ }^{12,13}$.

To overcome the drawbacks of lag screws, Herbert bone screws (HBS)were introduced as a variation of the traction osteosynthesis modality. Itcontains a differential pitch pattern of threads at both ends of the screw, giving the screw its compressive power. Furthermore, these screws are headless at both ends. The screw design was proposed by Herbert and Fisher in 1984to provide a rigid, safe and minimally invasive alternative that is used in the treatment of fractures of small bones of the hand ${ }^{14}$.

Despite that HBS osteosynthesis was only introduced recently as a new treatment modality for the management of mandibular fractures ${ }^{15,16}$, the clinical and radiographic behavior of solitary multiplaner HBS in MAFs treatment is insufficiently demonstrated in the contemporary literature. Accordingly, this study was designed to investigate the clinical and radiographic behavior of two different fixation modalities in the management of MAFs, where the outcome of the solitary multiplaner HBS is compared to the commonly utilized two miniplates on the tension and compression bands.

\section{PATIENTS AND METHODS}

A randomized, controlled, parallel-grouped clinical trial was conducted to compare the clinical and radiographic outcomes of solitary HBS and two 2.0-mm miniplates in the management of MAFs. Patientswere selected from the cases admitted to the Emergency Ward of Alexandria University Hospital.

Twenty adult patients with no gender predilection diagnosed with recent, uninfected, unilateral, non-comminuted, unfavorable isolated fracture in the angle region that demands open reduction and internal fixation were selected. Subjects with infected fracture lines, concomitant mandibular or other facial fractures, or a systemic disease that could cause interference with the fracture healing were excluded from this study. All participants in this study signed an informed consent before embarking on the surgical operation. 
According to the type of the utilized fixation device, the patients were randomly allocated into two groups. Patients in group I were treated using a solitary 2.3-mm HBS.Onthe other hand, two parallel miniplates with $2.0-\mathrm{mm}$ monocortical screws were used to manage patients in group II. Both fixation devices were from the same manufacturer (JEIL Medical Corporation Company: Seoul, Korea). The utilized HBS had a shaft diameter of $1.25 \mathrm{~mm}$, a cortical end diameter of $2.3 \mathrm{~mm}$ and a 2.0 cancellousend. The HBS kit comes with variable screw lengths, ranging from 10 to $30 \mathrm{~mm}$. The chosen length varied according to the case, and it was determined using KirschnerGuidewire (K-wire).

A detailed history taking and a thorough clinical examination were performed and recorded for every patient. A preoperative computerized tomogram (CT) scan (Ingenuity Core; Philips Medical Systems, Cleveland, $\mathrm{OH}$ ) was obtained to show the extent of fracture line, the degree and direction of displacement, and the relation of teeth involved in the fracture line. All patients received intravenous preoperative prophylactic antibiotic Amoxicillin $1000 \mathrm{mg}$ + Clavulanic acid $200 \mathrm{mg}$ (Augmentin 1.2 g; GlaxoSmithKline, UK).

After fracture exposure, a $0.8 \mathrm{~mm}$ K-Guide Wirewas placedperpendicular to and at least $1 \mathrm{~cm}$ away from the fracture line. The depth of the K-wire was measured using a depth gauge to determine the length of the screw to be utilized. A $2 \mathrm{~mm}$ cannulated spiral drill was used under the guidance of the K-Guide to make the osteotomy, followed by HBS placement, with the aid of the cannulated torque shank screwdriver (Figure 1). For patients in group II, two miniplates were contoured, applied and fixed using 2.0-mm monocortical screws, where a minimum of two screws were placed on either side of the fracture line.

At the end of surgery, the temporary IMF was released, the occlusion was checked and the surgical wound was sutured in layers. All patients received postoperative antibiotics in the form of Amoxicillin $500 \mathrm{mg}+$ Clavulanic acid $125 \mathrm{mg}$ (Augmentin 625 mg; GlaxoSmithKline, UK) orally three times per day for 5 days after discharge.

\section{Postoperative assessment}

A postoperative clinical evaluation was performed to observe any complications, which were classified into minor complications as Wound dehiscence, infection, and malocclusion, andmajor complications, as delayed union, non-union, sever interfragmentary mobility. The follow-up sessions were set after 1 week, 4 weeks, 6 weeks and 12 weeks. Radiographicappraisal was conducted using an immediate postoperative panoramic x-ray, followed by athree months CT-scanto show fracture healing and for radiodensitometric estimate of the mean bone density at the fracture line in comparison with the preoperative scan. A region of interest (ROI) around the fracture line was used to get the mean bone density using the CT-scan software. All the measurements were in Hounsfield Units $(\mathrm{HU})^{17}$.

\section{Statistical analysis}

All of the recorded data were documented, tabulated and statistically analyzed utilizing the IBM SPSS software package version 20.0. (Armonk, NY: IBM Corp).Differences between groups were analyzed and the significance of the obtained results was set at the $5 \%$ level.

\section{RESULTS}

Analysis of the study demographic data declared a male predilection $(75 \%, \mathrm{n}=15)$, with a male to female ratio of $3: 1$. The mean age of the patients was $28 \pm 3.9$ years. Road traffic accidents (RTA) was the most prevalent etiological factor $(50 \%, \mathrm{n}=10)$, followed by claimed falls $(35 \%, \mathrm{n}=7)$ and interpersonal violence (IPV) $(15 \%, n=3)$.

The study was performed on fifteen cases with right mandibular angle fracture and five patients 
with fractures in the left side.Twelvepatients $(60 \%)$ had a tooth present at the fracture line.There were no statistically significant differences between the groups concerning the demographic results (Table 1).

All of the studied cases were monitored postoperatively for three months.None of the cases showed signs of minor complications as neither infection nor wound dehiscence were encountered across the follow-up period. Furthermore, in both groups, normal occlusal and intercuspal relation was attained with no need for selective grinding in any case during the follow-up period.
Assessment of the interfragmentary mobility across the fracture line showed that there was no interfragmentarymobility in any of the cases in both groups.

A statistically significant gain in the mean bone density was found when comparing thethree months' postoperative CT-scan valuesto the preoperative estimates in both groups $(P<0.001)$. Cases treated with solitary HBS showed a higher percentage of increase in comparison to the preoperative values $(30.57 \pm 2.90 \%)$ than cases treated with miniplates $(29.74 \pm 5.15 \%)$. However, the difference was not statistically significant $(P=0.761)$ (Table 2).

TABLE (1) preoperative demographic data

\begin{tabular}{|c|c|c|c|}
\hline & HBS Group & MP Group & $P$-value \\
\hline Age, years, mean $\pm \mathrm{SD}$ & $27 \pm 3.4$ & $30 \pm 4.1$ & 0.677 \\
\hline \multicolumn{3}{|l|}{ Gender $(\%)$} & \multirow{4}{*}{1.000} \\
\hline Male & $80 \%(\mathrm{n}=8)$ & $70 \%(n=7)$ & \\
\hline Female & $20 \%(\mathrm{n}=2)$ & $30 \%(n=3)$ & \\
\hline Medical history & Free & Free & \\
\hline \multicolumn{3}{|l|}{ Trauma Etiology } & \multirow{4}{*}{0.473} \\
\hline Falls & $50 \%(\mathrm{n}=5)$ & $20 \%(n=2)$ & \\
\hline Road Traffic accidents & $40 \%(\mathrm{n}=4)$ & $60 \%(\mathrm{n}=6)$ & \\
\hline Interpersonal violence & $10 \%(\mathrm{n}=1)$ & $20 \%(n=2)$ & \\
\hline \multicolumn{3}{|l|}{ Fracture site } & \multirow{3}{*}{1.000} \\
\hline Left mandibular angle & $30 \%(n=3)$ & $20 \%(n=2)$ & \\
\hline Right mandibular angle & $70 \%(n=7)$ & $80 \%(\mathrm{n}=8)$ & \\
\hline
\end{tabular}

\section{SD, Standard Deviation}

TABLE (2) Mean bone density values

\begin{tabular}{|l|c|c|c|}
\hline CT-Scan intervals & HBS Group & MP group & P-value (t-test) \\
\hline Preoperative & $917.9 \pm 26.17$ & $919.1 \pm 31.20$ & 0.930 \\
\hline Three months postoperative & $1198.1 \pm 25.18$ & $1192.1 \pm 55.18$ & 0.761 \\
\hline Percentage of change & $30.57 \pm 2.90$ & $29.74 \pm 5.15$ & \\
\hline P-value(F-test) & $<0.001 *$ & $<0.001 *$ & \\
\hline
\end{tabular}

F test (ANOVA) with repeated measures for comparing between different follow-up period.

t- test for comparing between the two groups. 


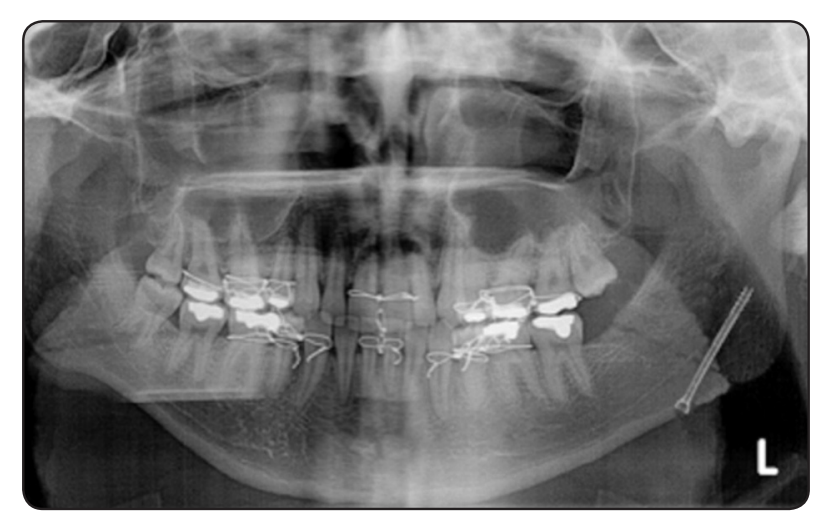

Fig. (1) Immediatepostoperative panoramic x-ray showing the inserted HBS.

\section{DISCUSSION}

Owing to the oblique nature of the fracture line in MAFs, several studies outlined the use of traction osteosynthesis in their management. These studieshave noted severaladvantages over bone plating, such as; the unsurpassed interfragmentary compression which nearly diminishes interfragmentary strains, the ability to provide a great functional stabilization along the entire length of the fracture line, and achieving a more anatomically accurate reduction. Moreover,rapid application and lower cost of the fixation device ${ }^{11,16,18}$. The holding power of traction osteosynthesis used for oblique fractures fixation depends on the cortical bone thickness, amount of the generated interfragmentary compression, and direction of screw placement ${ }^{19}$. Schaafet $\mathrm{al}^{11}$, compared the radiographic performance of solitary lag screw with that of double miniplates in the management of MAFs. Their study reported a significantly smaller postoperative fracture gap when using the lag screw ${ }^{11}$.

Herbert bone screw is recently adopted into the maxillofacial region. It is able to achieve great compression while at the same time it is safer on the thin bones by the virtue of its headless design ${ }^{14}$. This study intended to introduce a new treatment option that could be usedfor the management of MAFs.
Results of this study concerning the state of the postoperative occlusion showed that all the subjects in both groups exhibited a normal intercuspal centric occlusion relation. Schaaf et al ${ }^{11}$, came out with the same outcome. However, Kotrashettiand $\mathrm{Singh}^{18}$ register a $26.7 \%(\mathrm{n}=2)$ of cases where HBS was utilized with postoperative malocclusion that required elastic traction for 15 days to regain their premorbid occlusion ${ }^{18}$.

None of the cases developed any minor complication as wound dehiscence or infection. A similar outcome was reached in the study conducted by Kotrashetti and Singh when HBS was used ${ }^{18}$. The low infection rates in this studymay be contributed to the prophylactically administered antibiotics,strict aseptic operative conditions, and the postoperative antibiotic. Kotrashettiand Singh ${ }^{18}$ didn't encounter wound dehiscence while using HBS, which is in accordance with this study.

Across the follow-up period, both groups recordeda statistically significant increase in the mean bone density. HBS group recorded a higher percentage of change when comparing the three months' scan to the preoperative ones. However, the difference between groups was statistically insignificant. Traction osteosynthesis achieves a stable union between two bone fragments by their compression in the axial direction with the aid of screws. These modalities promote an unsurpassed rigidity and great compression across the entire length of the fracture line, providing an unparalleled rigidity compared to the rivaling fixation techniques ${ }^{12}$. Kotrashetti and Singh $^{18}$ used radiographicisodensity values from two-dimensional panoramic x-rays to announce that HBS achieves a faster bone healing than lag screw. Furthermore, their study design wasn't confined to the mandibular angle area ${ }^{18}$. In this study comparison between HBS and miniplates osteosynthesiswas performed only inmandibular angle area.

The headless feature of the screw, its reliance on the differential pitch pattern between its leading and 
trailing ends, and its self-tapping feature gives HBS distinct advantages over lag screw, especially forthe application in thin bones like that of the mandibular angle region. Unlike lag screw, HBS installation doesn't require countersinking, widening of the traction hole in the distal bony segment or screw pre-tapping. All along with minimizing the amount of drilling and reduction in the caliber of the osteotomy. HBS instillation eliminates the need for multiple drills pits which explain its accurate and minimally invasive placement technique $\mathrm{e}^{16,18,20}$.

The cannulated feature of the HBS allows its utilization along with the K-Guide wire, which prompts a more predictable and minimally invasive device placement. This technique allows the use of depth gage to accurately determine the depth and length of the osteotomy, gives an arbitrary verification about the integrity of the surrounding bone cortices, and a primary insight about the final screw position. Furthermore, drilling on top of the guide wire using a cannulated spiral drill favors a smaller caliper of the drill to be used, with reduction of off-centric rotation side effect of the drill, thus minimizing the diameter of the osteotomy ${ }^{21}$. Owing to the utilization of K-Guide wire in this study, none of the cases experienced any breach of the lateral or medial cortices of the ramus. This was confirmed by the axial and coronal cuts in the postoperative $\mathrm{x}$-rays.

The placement of a solitary multi-planer HBS can achieve a predictable interfragmentary compression along the entire length of the fracture line. This treatment modality is comparable with the most rigid type of the non-compressive bone platting modalities, with the added leverage of attaining interfragmentary compression, reducing steps of hardware installation, and minimizing the amount of fixation material used. Furthermore, standardizing the same manufacturer for the two treatment modalities revealed that solitary HBS was much cheaper than double miniplates.On the other hand, it is a technique sensitive treatment modality that requires surgical expertise.

The lack of significant differences between the clinical and radiographic performances of the HBS when compared to the double miniplate fixation may point out the novelty of the treatment modality in the maxillofacial trauma field. In order to validate the outcomes reached by this study, further prospective studies with larger sample size are required.

\section{CONCLUSION}

Bringing the study limitation into consideration, it is possible to conclude that HBS offers a rigid, functionally stable, more economic, and predictable treatment option for the management of MAFs.

\section{REFERENCES}

1. Jadhav A, Mundada B, Deshmukh R, Bhutekar U, Kala A, Waghwani K, et al. Mandibular Ramus Fracture: An Overview of Rare Anatomical Subsite. Plast Surg Int;954314, 2015.

2. Gassner R, Tuli T, Hächl O, Rudisch A, Ulmer H. Craniomaxillofacial trauma: a 10 year review of 9,543 cases with 21,067 injuries. J Craniomaxillofac Surg; 31 (1):5161,2003 .

3. Stacey DH, Doyle JF, Mount DL, Snyder MC, Gutowski KA. Management of mandible fractures. Plast Reconstr Surg; 117 (3):48e-60e, 2006.

4. Zimmermann C, Henningsen A, Henkel KO, Klatt J, Jürgens C, Seide K, et al. Biomechanical comparison of a multidirectional locking plate and conventional plates for the osteosynthesis of mandibular angle fractures-A preliminary study. J Craniomaxillofac Surg; 45 (12):1913-1920, 2017.

5. Champy M, Loddé JP, Schmitt R, Jaeger JH, Muster D. Mandibular osteosynthesis by miniature screwed plates via a buccal approach. J Maxillofac Surg; 6 (1):14-21, 1978.

6. Kolk A, Neff A. Long-term results of ORIF of condylar head fractures of the mandible: A prospective 5-year follow-up study of small-fragment positional-screw osteosynthesis (SFPSO). J Craniomaxillofac Surg; 43(4):452461, 2015. 
7. Guimond C, Johnson JV, Marchena JM. Fixation of mandibular angle fractures with a 2.0-mm 3-dimensional curved angle strut plate. J Oral Maxillofac Surg; 63 (2):209-214, 2005.

8. Ellis E. A prospective study of 3 treatment methods for isolated fractures of the mandibular angle. J Oral Maxillofac Surg; 68 (11):2743-2754, 2010.

9. Monnazzi MS, Gabrielli MA, Gabrielli MF, Trivellato AE. Mandibular angle fractures: a comparative study between one- and two-plate fixation. Dent Traumatol; 33 (2):121125, 2017.

10. Brons R, Boering G. Fractures of the mandibular body treated by stable internal fixation: a preliminary report. J Oral Surg; 28 (6):407-415, 1970.

11. Schaaf H, Kaubruegge S, Streckbein P, Wilbrand JF, Kerkmann H, Howaldt HP. Comparison of miniplate versus lagscrew osteosynthesis for fractures of the mandibular angle. Oral Surg Oral Med Oral Pathol Oral Radiol Endod; 111 (1):34-40, 2011.

12. Ellis E. Is lag screw fixation superior to plate fixation to treat fractures of the mandibular symphysis? J Oral Maxillofac Surg; 70 (4):875-882, 2012.

13. Falci SG, Rodrigues DC, Marchiori EC, Brancher G, Makyama A, Fernandes Moreira RW. Assessment of the fixation of mandibular symphysis fractures using conical cannulated screws: mechanical and photoelastic tests. Oral Surg Oral Med Oral Pathol Oral Radiol; 118 (2):174-180, 2014.

14. Alexa O, Veliceasa B. Percutaneous Herbert screw osteosynthesis in trans-scaphoid perilunate fracture-dislocations. Rev Med Chir Soc Med Nat Iasi2013; 117 (2): 409-413.
15. Feichtinger $M$, Schultes $G$, Kärcher $H$. The use of a $3 D$ navigation system in the treatment of mandibular angle fractures by minimally invasive insertion of Herbert screws for osteosynthesis. Comput Aided Surg; 13 (1):47$54,2008$.

16. Wallner J, Reinbacher K, Feichtinger M, Pau M, Feigl G, Quehenberger F, et al. Osteosynthesis using cannulated headless Herbert screws in mandibular angle fracture treatment: A new approach? J Craniomaxillofac Surg; 45 (4):526-539, 2017.

17. Shapurian T, Damoulis PD, Reiser GM, Griffin TJ, Rand WM. Quantitative evaluation of bone density using the Hounsfield index. Int $\mathbf{J}$ Oral Maxillofac Implants; 21 (2):290-297, 2006.

18. Kotrashetti SM, Singh AG. Prospective study of treatment outcomes with lag screw versus Herbert screw fixation in mandibular fractures. Int J Oral Maxillofac Surg; 46 (1):54-58, 2017.

19. Ehlinger M, Czekaj J, Adam P, Brinkert D, Ducrot G, Bonnomet F. Minimally invasive fixation of type B and C interprosthetic femoral fractures. Orthop Traumatol Surg Res; 99 (5):563-569, 2013.

20. Park JW, Kim KT, Sung JK, Park SH, Seong KW, Cho DC. Biomechanical Comparison of Inter-fragmentary Compression Pressures: Lag Screw versus Herbert Screw for Anterior Odontoid Screw Fixation. J Korean Neurosurg Soc; 60 (5):498-503, 2017.

21. Hsu LP, Schwartz EG, Kalainov DM, Chen F, Makowiec RL. Complications of $\mathrm{K}$-wire fixation in procedures involving the hand and wrist. J Hand Surg Am; 36 (4):610$616,2011$. 